\title{
Virus and bacterium gang up on host
}

\section{ce \\ exposure to virus-related compounds altered the macrophage response to bacterial MDP}

g

Infectious diseases caused by viruses such as influenza viruses are often followed by bacterial infection. Separately, these infections are not necessary lethal, but their combination results in higher mortality levels. Writing in a recent issue of Cell Host \& Microbe, Gabriel Núñez and colleagues now describe an immunological mechanism by which viral infections can heighten the immune response to a subsequent bacterial infection, leading to increased mortality.

Cells detect invading pathogens through various innate defences. RNA viruses are recognized in part by RNA sensors, such as RIG-I (also known as DDX58) and MDA5 (also known as IFIH1), in the host cytosol, or by Toll-like receptor 3 , leading to an antiviral response that includes the production of type I interferons (IFNs). By contrast, bacterial peptidoglycan is recognized by the NOD-like receptors NOD1 and NOD2. To determine how the antiviral response affects the antibacterial

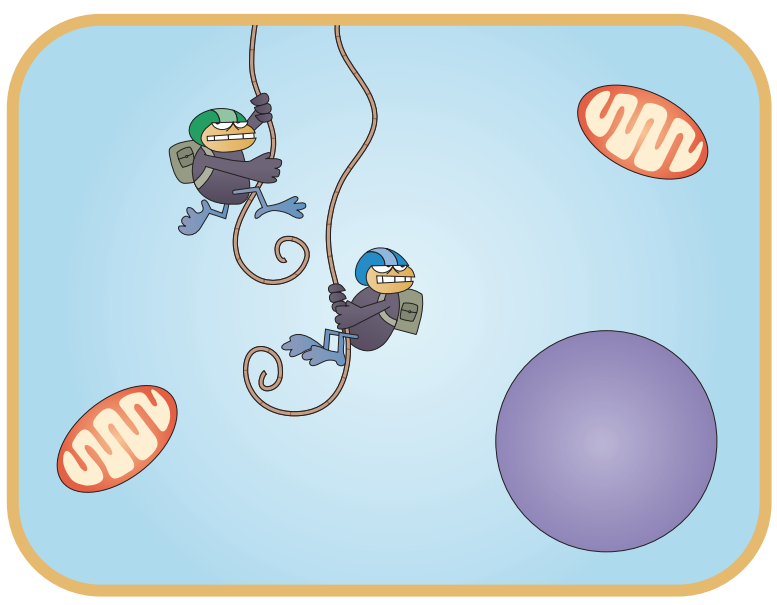

response and subsequent disease outcome, the authors simulated viral and bacterial infections in mouse bone marrow macrophages with the double-stranded RNA molecule polyinosinic-polycytidylic acid (polyI:C) and muramyl dipeptide (MDP), respectively, and determined how this affected activation of mitogen-activated protein kinases (MAPKs) and nuclear factor- $\kappa \mathrm{B}$ (NF- $\kappa \mathrm{B})$, two important transcriptional regulators of immune responses. There was little change in MAPK and NF- $\kappa$ B activation after treatment with either polyI:C or MDP. However, levels of MAPK and NF- $\kappa B$ activation increased sharply in cells treated with polyI:C prior to MDP addition. Furthermore, levels of the pro-inflammatory molecules interleukin-6 (IL-6) and tumour necrosis factor (TNF) were higher after treatment with polyI:C and MDP than after treatment with MDP alone, an effect that required NOD2. Thus, exposure to virus-related compounds altered the macrophage response to bacterial MDP.

As polyI:C can induce the production of type I IFNs, the authors speculated that this could be the link between the antiviral and antibacterial responses. Indeed, the heightened response to MDP after polyI:C treatment was not detected in macrophages lacking the type I IFN receptor. Furthermore, type I IFN induced the expression of NOD1, NOD2 and RIP2 (also known as RIPK2; a NOD response adaptor).

To determine whether these processes are responsible for the increased lethality of a secondary bacterial infection in vivo, wild-type mice and mice lacking both NOD1 and NOD2 were infected with Escherichia coli following treatment with norovirus, polyI:C or PBS. All PBS-treated mice survived the bacterial infection. Among the animals that had been pretreated with virus or polyI:C, Nod1 $1^{-/-} \mathrm{Nod}_{2}^{-/-}$mice were killed by the bacterial infection at a lower rate than similarly treated wildtype mice. Thus, a norovirus infection can increase the lethality of a bacterial infection in vivo in a NOD1- and NOD2-dependent manner, although the remaining lethality in the $\mathrm{Nod}^{-/-}$ Nod2 $2^{-1-}$ mice indicates that additional factors may potentiate the lethality of the secondary bacterial infection.

The authors propose that recognition of viral RNA by cytosolic or membrane-bound RNA sensors induces the production of type I IFNs, which stimulate the production of NOD1, NOD2, RIP2 and possibly other factors. The increased levels of these factors then heighten the response to bacterial products, leading to the secretion of high levels of IL-6 and TNF. Ultimately, the high levels of these pro-inflammatory molecules can promote organ failure and have lethal effects. Insight into the crosstalk between the antiviral and antibacterial responses may provide leads on interventions that can prevent the high lethality of these secondary infections.

Christiaan van Ooij

ORIGINAL RESEARCH PAPER Kim, Y.-G., et al. Viral infection augments Nod $1 / 2$ signaling to potentiate lethality associated with secondary bacterial infections. Cell Host Microbe 9, 496-507 (2011) 